\title{
Kerov's central limit theorem for Schur-Weyl and Gelfand measures (extended abstract)
}

\author{
Pierre-Loïc Méliot t| \\ Institut Gaspard Monge — Université Paris-Est Marne-La-Vallée — Marne-La-Vallée — France
}

\begin{abstract}
We show that the shapes of integer partitions chosen randomly according to Schur-Weyl measures of parameter $\alpha=1 / 2$ and Gelfand measures satisfy Kerov's central limit theorem. Thus, there is a gaussian process $\Delta$ such that under Plancherel, Schur-Weyl or Gelfand measures, the deviations $\Delta_{n}(s)=\lambda_{n}(\sqrt{n} s)-\sqrt{n} \lambda_{\infty}^{*}(s)$ converge in law towards $\Delta(s)$, up to a translation along the $x$-axis in the case of Schur-Weyl measures, and up to a factor $\sqrt{2}$ and a deterministic remainder in the case of Gelfand measures. The proofs of these results follow the one given by Ivanov and Olshanski for Plancherel measures; hence, one uses a "method of noncommutative moments".

Résumé. Nous montrons que les formes des partitions d'entiers choisies aléatoirement sous les mesures de SchurWeyl de paramètre $\alpha=1 / 2$ et sous les mesures de Gelfand obéissent au théorème central limite de Kerov. Ainsi, il existe un processus gaussien $\Delta$ tel que sous les mesures de Plancherel, de Schur-Weyl ou de Gelfand, les déviations $\Delta_{n}(s)=\lambda_{n}(\sqrt{n} s)-\sqrt{n} \lambda_{\infty}^{*}(s)$ convergent en loi vers $\Delta(s)$, à une translation près le long de l'axe des abscisses pour les mesures de Schur-Weyl, et à un facteur $\sqrt{2}$ et un reste déterministe près dans le cas des mesures de Gelfand. Les preuves de ces résultats suivent celle donnée par Ivanov et Olshanski pour les mesures de Plancherel; ainsi, on utilise une "méthode de moments non commutatifs".
\end{abstract}

Keywords: Random partitions, representation theory of symmetric groups.

In this article, we investigate the fluctuations of random partitions chosen according to probability measures stemming from the representation theory of the symmetric groups. Given a group $G$ and a (complex, finite-dimensional) representation $V$ of $G$, the decomposition of $V$ in irreducible components yields a probability measure $\mathbb{P}_{V}$ on the set $\widehat{G}$ of isomorphism classes of irreducible representations of $G$ :

$$
V=\bigoplus_{\lambda \in \widehat{G}} m_{\lambda} V^{\lambda} \Rightarrow \mathbb{P}_{V}[\lambda]=\frac{m_{\lambda} \operatorname{dim} V^{\lambda}}{\operatorname{dim} V}
$$

When $G=\mathfrak{S}_{n}$ is the symmetric group of size $n$, the elements of $\widehat{\mathfrak{S}}_{n}$ can be labelled by integer partitions of size $n$, that is to say, non-increasing sequences of positive integers that sum to $n$; see $e . g$. [Ful97]. We shall denote by $\mathfrak{P}_{n}$ the set of partitions of size $n$, and a partition $\lambda=\left(\lambda_{1}, \lambda_{2}, \ldots, \lambda_{r}\right)$ will be represented by its Young diagram, that is the array of $n$ boxes with $\lambda_{1}$ boxes on the first row, $\lambda_{2}$ boxes on the second

†meliotephare.normalesup.org

1365-8050 @ 2011 Discrete Mathematics and Theoretical Computer Science (DMTCS), Nancy, France 
row, etc. Hence, any representation $V_{n}$ of $\mathfrak{S}_{n}$ yields a model of random Young diagrams in $\mathfrak{P}_{n}$. For various families of representations $\left(V_{n}\right)_{n \in \mathbb{N}}$, one can establish limit theorems for the shapes of the Young diagrams chosen randomly according to the measures $\mathbb{P}_{V_{n}}$. In particular, let us consider the case when $V_{n}=\mathbb{C S}_{n}$ is the (left) regular representation of $\mathfrak{S}_{n}$; then, $m_{\lambda}=\operatorname{dim} V^{\lambda}$ for any partition $\lambda$, and $\mathbb{P}_{n}[\lambda]=\left(\operatorname{dim} V^{\lambda}\right)^{2} / n$ !. The following result has been proved by Logan, Shepp, Kerov and Vershik, and a complete exposition can be found in [IO02]:

Theorem 1 Let $\lambda_{n}$ be a random partition of size $n$ under the Plancherel measure $\mathbb{P}_{n}=\mathbb{P}_{\mathbb{C S}_{n}}$; we denote $s \mapsto \lambda_{n}(s)$ the upper boundary of the Young diagram rotated $45^{\circ}\left(\right.$ see $\$ 7$, and $\lambda_{n}^{*}(s)=\lambda_{n}(s \sqrt{n}) / \sqrt{n}$. As $n$ goes to infinity, $\lambda_{n}^{*}$ converges in probability and for the topology of uniform convergence on $\mathbb{R}$ towards the function

$$
\lambda_{\infty}^{*}(s)=\Omega(s)= \begin{cases}\frac{2}{\pi}\left(s \arcsin \left(\frac{s}{2}\right)+\sqrt{4-s^{2}}\right) & \text { if }|s|<2 \\ |s| & \text { if }|s| \geq 2 .\end{cases}
$$

Moreover, on $[-2,2], \Delta_{n}(s)=\sqrt{n}\left(\lambda_{n}^{*}(s)-\Omega(s)\right)$ converges in law towards the generalized gaussian process

$$
\Delta(s)=\frac{2}{\pi} \sum_{k \geq 2} \frac{\xi_{k}}{\sqrt{k}} \sin (k \theta) \quad \text { with } s=2 \cos \theta
$$

where the $\xi_{k}$ 's are standard independent gaussian variables.

The purpose of this paper is to describe a similar result of gaussian concentration for two other models of random partitions: the Schur-Weyl measures and the Gelfand measures ( $c f . \$ 1$. The limit shapes $\lambda_{\infty}^{*}$ for these models have already been computed, see for instance [Bia01] and [LS77]; so the true novelty of this paper consists in the central limit theorems, see Theorems 2 and 5 . The situation can be summarized as follows:

- For Schur-Weyl measures $\mathbb{S W}_{n, c}$, everything happens as if the fluctuations $\sqrt{n}\left(\lambda_{n}^{*}(s)-\lambda_{\infty}^{*}(s)\right)$ were those of the Plancherel measures, up to a translation of $c$ along the $x$-axis.

- Similarly, for Gelfand measures $\mathbb{G}_{n}$, the fluctuations are again those of the Plancherel measures, but this time multiplied by a factor $\sqrt{2}$ and translated by a deterministic function along the $y$-axis.

Hence, the same generalized gaussian process $\Delta$ is involved in the description of the fluctuations of the random shapes under Schur-Weyl and Gelfand measures; this is a striking fact, and our main result.

To prove this, we shall follow and adapt the proof of Theorem 1 given by Ivanov and Olshanski in [IO02]; it is essentially a method of moments, and it involves the algebra of observables of diagrams, see \$2. By using Śniady's theory of cumulants of observables, we shall first prove that irreducible characters $\chi^{\lambda}\left(k 1^{n-k}\right)$ under Schur-Weyl or Gelfand measures are jointly asymptotically gaussian ( $\$ 3$, and we shall compute explicitly their limit laws. Then, we will use exactly as in [IO02] the combinatorics of Chebyshev polynomials of the second kind to deduce from the asymptotics of characters the asymptotics of the shapes of the random partitions. Although most of our arguments can be found in the two papers [IO02, S06], new difficulties have arised in our asymptotic study. Thus, for Schur-Weyl measures, the calculations of linear functionals of the fluctuations involve hypergeometric identities; whereas for Gelfand measures, 
the problem resides mainly in the proof of the joint convergence of the renormalized character values, and one has to go beyond the setting of asymptotic factorization described in [Ś06], see Lemma 4

This paper should be thought of as a extended abstract of [Mél10a] and [Mél10b]. In particular, we only present sketches of proofs, and we will omit most of the technical computations (for instance, the aforementioned hypergeometric identities in the case of Schur-Weyl measures). The author would like to thank M. Sage for showing him a proof of the second part of Lemma 4 and P. Biane and V. Féray for various comments and suggestions.

\section{Schur-Weyl and Gelfand measures}

A first family of representations of the symmetric groups is provided by tensor powers of vector spaces. If $N$ is an integer, let us consider the space $W=\left(\mathbb{C}^{N}\right)^{\otimes n}$; it is endowed with a "diagonal" action of $\operatorname{GL}(N, \mathbb{C})$ on the left $g \cdot\left(v_{1} \otimes v_{2} \otimes \cdots \otimes v_{n}\right)=g\left(v_{1}\right) \otimes g\left(v_{2}\right) \otimes \cdots \otimes g\left(v_{n}\right)$, and with an action of $\mathfrak{S}_{n}$ on the right by permutation of the letters: $\left(v_{1} \otimes v_{2} \otimes \cdots \otimes v_{n}\right) \cdot \sigma=v_{\sigma(1)} \otimes v_{\sigma(2)} \otimes \cdots \otimes v_{\sigma(n)}$. The algebras generated by these two actions are mutual full centralizers in $\operatorname{End}(W)$, whence a decomposition of $W$ in direct sum of irreducible $\left(\mathrm{GL}(N, \mathbb{C}), \mathfrak{S}_{n}\right)$-bimodules:

$$
\left(\mathbb{C}^{N}\right)^{\otimes n}=\bigoplus_{\substack{\lambda \in \mathfrak{P}_{n} \\ \ell(\lambda) \leq N}} H^{\lambda}(N) \otimes_{\mathbb{C}} S^{\lambda}
$$

Here, $H^{\lambda}(N)$ is the irreducible $\operatorname{GL}(N, \mathbb{C})$-module of highest weight $\lambda$, and $S^{\lambda}$ denotes the irreducible Specht module of $\mathfrak{S}_{n}$ of type $\lambda$. The Schur-Weyl measure of parameters $N$ and $n$ is the measure on partitions of size $n$ and length less than $N$ associated to this decomposition:

$$
\mathbb{P}_{N, n}[\lambda]=\frac{\operatorname{dim} H^{\lambda}(N) \times \operatorname{dim} S^{\lambda}}{N^{n}}=\left(\prod_{\square \in \lambda} 1+\frac{c(\square)}{N}\right) \times \frac{n !}{\prod_{\square \in \lambda} h(\square)^{2}}
$$

where $\{\square \in \lambda\}$ is the set of boxes of the Young diagram $\lambda, c(i, j)=i-j$ is the content of a box $\square=(i, j)$, and $h(i, j)=\left(\lambda_{j}-i\right)+\left(\lambda_{i}^{\prime}-j\right)+1$ is the hook length of the box, see [Ful97] for a proof of this formula. Since the term $n ! /\left(\prod_{\square \in \lambda} h(\square)^{2}\right)$ is precisely the Plancherel measure $\mathbb{P}_{n}[\lambda]$, Schur-Weyl measures can be thought of as deformations of the Plancherel measures (fix $n$ and let $N$ go to infinity). With this point of view, an interesting scale to look at is when $\sqrt{n} \simeq c N$ with $c \geq 0$; with a slight abuse of notation ${ }^{(i)}$, we shall denote $\mathbb{S W}_{n, c}$ the corresponding Schur-Weyl measure.

If $g \in \mathrm{GL}(N, \mathbb{C})$ has eigenvalues $x_{1}, \ldots, x_{N}$ and $\sigma \in \mathfrak{S}_{n}$ has cycle type $\mu \in \mathfrak{P}_{n}$, it is not very difficult to show that the bitrace of $(g, \sigma)$ acting on $\left(\mathbb{C}^{N}\right)^{\otimes n}$ is given by the symmetric function $p_{\mu}\left(x_{1}, \ldots, x_{N}\right)$. As a consequence, if one denotes by $\chi^{\lambda}$ (respectively, $\varsigma^{\lambda}$ ) the normalized (resp., non normalized) irreducible character of $\mathfrak{S}_{n}$ of type $\lambda$, and if $\lambda$ is picked randomly according to the Schur-Weyl measure, then for any permutation $\sigma$ of type $\mu$ :

$$
\mathbb{S W}_{n, c}\left[\chi^{\lambda}(\sigma)\right]=\frac{1}{N^{n}} \sum_{\lambda} \operatorname{dim} H^{\lambda}(N) \times \varsigma^{\lambda}(\sigma)=\frac{\operatorname{bitr}(\mathrm{id}, \sigma)}{N^{n}}=\frac{p_{\mu}\left(1^{N}\right)}{N^{n}}=N^{\ell(\mu)-|\mu|}
$$

\footnotetext{
(i) Indeed, the Schur-Weyl measure depends on the exact value of $N$, but for our asymptotic study, the estimate $\sqrt{n} \simeq c N$ will be sufficient.
} 
Notice that the quantity $|\mu|-\ell(\mu)$ is not changed if one adds or removes parts of size 1 to the integer partition $\mu$. In the following, we shall rather work with renormalized character values $\Sigma_{\mu}(\lambda)$, that are defined in the following way for any pair of partitions $(\lambda, \mu)$ of respective sizes $n$ and $k$ :

$$
\Sigma_{\mu}(\lambda)= \begin{cases}n(n-1) \cdots(n-k+1) \chi^{\lambda}\left(\mu \sqcup 1^{n-k}\right)=n^{\downarrow|\mu|} \chi^{\lambda}\left(\mu \sqcup 1^{n-|\mu|}\right) & \text { if } n \geq k, \\ 0 & \text { otherwise. }\end{cases}
$$

With these conventions, and assuming that $\sqrt{n}=c N+O(1)$, Equation (6) becomes:

$$
\mathbb{S W}_{n, c}\left[\Sigma_{\mu}\right]=c^{|\mu|-\ell(\mu)} n^{\frac{|\mu|+\ell(\mu)}{2}}\left(1+O\left(n^{-1 / 2}\right)\right) .
$$

This identity will turn out to be fundamental for the asymptotic analysis of Schur-Weyl measures.

If $G$ is a finite group, recall that a Gelfand model for $G$ is a representation in which each irreducible representation of $G$ appears exactly one time. The Gelfand models of the symmetric groups have been studied for instance in [APR08], and they provide another interesting family of probability measures on the sets $\mathfrak{P}_{n}$, which we shall call Gelfand measures and denote by $\mathbb{G}_{n}$ :

$$
\mathbb{G}_{n}[\lambda]=\frac{\operatorname{dim} S^{\lambda}}{\sum_{\mu \in \mathfrak{P}_{n}} \operatorname{dim} S^{\mu}}=\frac{\operatorname{dim} S^{\lambda}}{I_{n}}
$$

The normalization constant $I_{n}$ is actually equal to the number of involutions of size $n$. More generally, the trace of a permutation $\sigma$ in a Gelfand model $G_{n}$ of $\mathfrak{S}_{n}$ is the number of "square roots" of $\sigma$, see Theorem 3.1 in [APR08]. As a consequence, for any permutation $\sigma$,

$$
\mathbb{G}_{n}\left[\chi^{\lambda}(\sigma)\right]=\frac{\operatorname{card}\left\{\tau \in \mathfrak{S}_{n} \mid \sigma=\tau^{2}\right\}}{\operatorname{card}\left\{\tau \in \mathfrak{S}_{n} \mid \operatorname{id}_{\llbracket 1, n \rrbracket}=\tau^{2}\right\}} .
$$

The number $I_{n}$ of involutions of size $n$ is well known to be asymptotically equivalent to $\left(\frac{n}{\mathrm{e}}\right)^{\frac{n}{2}} \frac{\mathrm{e}^{\sqrt{n}-1 / 4}}{\sqrt{2}}$ - this follows from a saddle point analysis of the exponential generating function $\exp \left(x+x^{2} / 2\right)$. On the other hand, for any partition $\mu$, one can give an exact formula for the number of square roots of a permutation of cycle type $\mu \sqcup 1^{n-|\mu|}$, see Corollary 3.2 in [APR08]. Using these two facts and [10], one concludes that for any partition $\mu=1^{m_{1}} 2^{m_{2}} \cdots s^{m_{s}}$ :

$$
\mathbb{G}_{n}\left[\Sigma_{\mu}\right]=\left(\prod_{i=2}^{s} f\left(i, m_{i}\right)\right) n^{\frac{|\mu|+m_{1}(\mu)}{2}}\left(1+O\left(n^{-1 / 2}\right)\right),
$$

where $f$ is the function on pairs of non-negative integers defined by:

$$
f(i, m)= \begin{cases}0 & \text { if } i \text { is even and } m \text { is odd } \\ \frac{m !}{m / 2 !}\left(\frac{i}{2}\right)^{m / 2} & \text { if } i \text { and } m \text { are even } \\ \sum_{k=0}^{\left\lfloor\frac{m}{2}\right\rfloor} \frac{m !}{m-2 k ! k !}\left(\frac{i}{2}\right)^{k} & \text { if } i \text { is odd. }\end{cases}
$$

Again, (11] is fundamental for the asymptotic analysis of Gelfand measures. 
If $\lambda$ is a Young diagram with longest rows at the bottom and boxes of area 2, let us rotate the figure $45^{\circ}$ counterclockwise, and consider the upper boundary $s \mapsto \lambda(s)$ of the planar shape - this is the usual russian convention for drawing Young diagrams. One obtains thus a Lipschitz function with constant 1 , such that $\lambda(s)=|s|$ for $|s|$ big enough. The scaled version $\lambda^{*}(s)=\lambda(s \sqrt{n}) / \sqrt{n}$ of this function is normalized so that $\int_{\mathbb{R}} \frac{\lambda^{*}(s)-|s|}{2} d s=1$. We have drawn below $\lambda^{*}(s)$ when $\lambda$ is a random partition of size $n=500$ under the Schur-Weyl measure of parameter $c=1$ (resp., under the Gelfand measure).

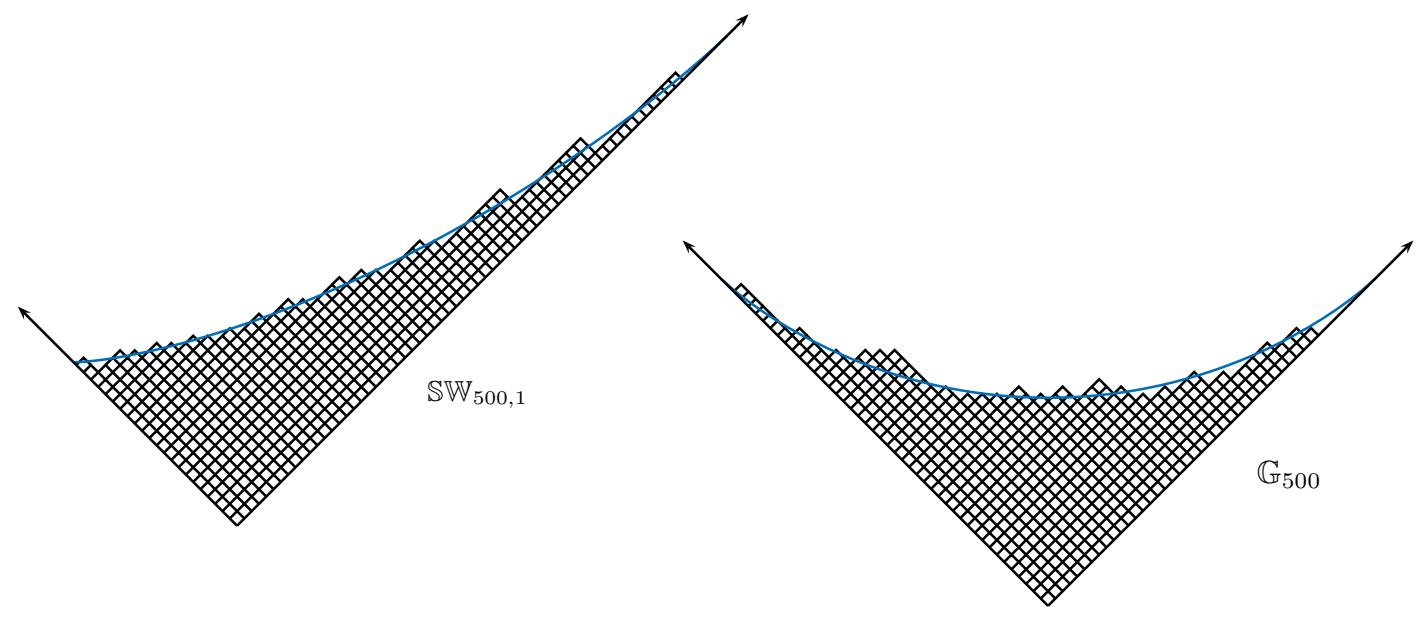

It is clear from the drawings that the scaled random partitions under Schur-Weyl measures $\mathbb{S W}_{n, c}$ and Gelfand measures $\mathbb{G}_{n}$ should have limit shapes $\Omega_{c}$ and $\Omega$ (figured in blue above). As we shall see hereafter, it is indeed the case, and $\Omega_{0}=\Omega$ is the limit shape evoked in Theorem 1 , the $\Omega_{c \neq 0}$ 's are some deformations of this curve. In the following, we will mainly be interested in the scaled fluctuations:

$$
\begin{array}{ll}
\Delta_{n, c}(s)=\sqrt{n}\left(\lambda^{*}(s)-\Omega_{c}(s)\right) & \text { with } \lambda \sim \mathbb{S W}_{n, c} \\
\Delta_{n}(s)=\sqrt{n}\left(\lambda^{*}(s)-\Omega(s)\right) & \text { with } \lambda \sim \mathbb{G}_{n}
\end{array}
$$

and in $\$ 4$ we will give a central limit theorem for these quantities.

To conclude this section, let us give some extra motivation for the asymptotic study of Schur-Weyl and Gelfand measures. First of all, recall that if $w$ is a word of size $n$ over an alphabet $\llbracket 1, A \rrbracket$, then the RSK algorithm ( $c f$. [Ful97]) associates to $w$ a pair of Young tableaux $(P(w), Q(w))$ such that $P(w)$ is semistandard with entries in $\llbracket 1, A \rrbracket, Q(w)$ is standard with entries in $\llbracket 1, n \rrbracket$, and $P(w)$ and $Q(w)$ have same shape $\lambda(w) \in \mathfrak{P}_{n}$. Moreover, the first rows (resp., the first columns) of $\lambda(w)$ correspond to the lengths of the longest non-decreasing (resp., decreasing) subwords in $w$. That said, the Schur-Weyl measure of parameters $N$ and $n$ (resp., the Gelfand measure of parameter $n$ ) is exactly the image by $w \mapsto \lambda(w)$ of the uniform measure on words of size $n$ over $\llbracket 1, N \rrbracket$ (resp., of the uniform measure on involutions of size $n$ ). Consequently, our results can be restated in asymptotic combinatorial properties of words. Another motivation comes from random matrix theory: indeed, models of random partitions can be considered as discrete analogues of models of random matrices, and in this correspondence, Gelfand measures are related to the GOE and Wigner's law (see [BR01]), and Schur-Weyl measures are related 
to random covariance matrices and Marčenko-Pastur laws ( $c f$. [Bia01]). Hence, asymptotic results on Gelfand and Schur-Weyl measures may provide a better understanding of some models from random matrix theory.

\section{Observables of diagrams and partial permutations}

If $\lambda$ is a Young diagram, let us denote by $x_{1}<y_{1}<x_{2}<y_{2}<\cdots<y_{v-1}<x_{v}$ the interlacing sequences of local mimina and local maxima of the function $s \mapsto \lambda(s)$. The interlacing moments $\widetilde{p}_{k \geq 1}(\lambda)$ of $\lambda$ are defined by:

$$
\widetilde{p}_{k}(\lambda)=\sum_{i=1}^{v}\left(x_{i}\right)^{k}-\sum_{i=1}^{v-1}\left(y_{i}\right)^{k}=\int_{\mathbb{R}} s^{k} \sigma_{\lambda}^{\prime \prime}(s) d s \quad \text { with } \sigma_{\lambda}(s)=\frac{\lambda(s)-|s|}{2} .
$$

The integral expression of $\widetilde{p}_{k}$ enables us to consider interlacing moments of more general planar shapes, for instance continuous diagrams, that is to say functions $s \mapsto \omega(s)$ such that $\omega$ is Lipschitz with constant 1 and equal to $|s|$ for $|s|$ big enough. On the other hand, $\widetilde{p}_{1}(\lambda)=0$ for any (continuous) Young diagram, and the $\widetilde{p}_{k \geq 2}$ 's generate a complex algebra of observables of diagrams which we shall denote $\mathscr{O}$, and which is freely generated by these $\widetilde{p}_{k}$ 's:

$$
\mathscr{O}=\mathbb{C}\left[\widetilde{p}_{2}, \widetilde{p}_{3}, \widetilde{p}_{4}, \ldots\right]
$$

In [IO02], it is shown that $\mathscr{O}$ also contains the symbols $\Sigma_{\mu}$ introduced in $\S 1$, and that $\mathscr{O}$ is freely generated by $\left(\Sigma_{k}\right)_{k \geq 1}$, and linearly generated by $\left(\Sigma_{\mu}\right)_{\mu \in \mathfrak{P}}$. Moreover, $\mathscr{O}$ is graded either by the weight $\operatorname{wt}\left(\Sigma_{\mu}\right)=$ $|\mu|+\ell(\mu)$ or by Kerov's degree $\operatorname{deg}_{K}\left(\Sigma_{\mu}\right)=|\mu|+m_{1}(\mu)$, and with respect to these gradations, $\widetilde{p}_{k \geq 2}$ writes as:

$$
\begin{aligned}
& \widetilde{p}_{k}=\sum_{\substack{\mu=1^{m_{1}} \\
|\mu|+\ell(\mu)=k}} \frac{k^{\downarrow \ell(\mu)}}{\prod_{i \geq 1} m_{i} !} \prod_{i \geq 1}\left(\Sigma_{i}\right)^{m_{i}}+\left\{\begin{array}{c}
\text { observable of weight } \\
\text { smaller than } k-1
\end{array}\right\}
\end{aligned}
$$

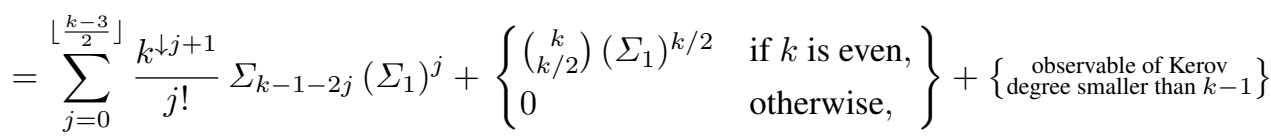

see [IO02, Proposition 3.7 and Equation 7.9]. Notice that by a change of basis between the $\widetilde{p}$ s and the $\Sigma$ 's, it is possible to evaluate the symbols $\Sigma_{\mu}$ on any continuous Young diagram $\omega$.

In the following, we will need to know how to compute a product $\Sigma_{\mu} \Sigma_{\nu}$ and decompose it in the basis $\left(\Sigma_{\lambda}\right)_{\lambda \in \mathfrak{P}}$; in other words, we ask for the structure constants of $\mathscr{O}$ with respect to the basis of rescaled character values. Although there is no general formula for these coefficients, many things can be said about the symbols $\Sigma_{\mu}$ if one interprets them as elements of the algebra of partial permutations, see [IK99] for the definition of these objects. Thus, if one associates to $\Sigma_{\left(\mu_{1}, \ldots, \mu_{r}\right)}$ the formal sum of partial permutations

$$
\sum_{a_{11} \neq a_{12} \neq \cdots \neq a_{r \mu_{r}}}\left[\left(a_{11}, \ldots, a_{1 \mu_{1}}\right) \cdots\left(a_{r 1}, \ldots, a_{r \mu_{r}}\right),\left\{a_{i j}\right\}_{1 \leq i \leq r, 1 \leq j \leq \mu_{i}}\right]
$$


then one gets an isomorphism between $\mathscr{O}$ and a commutative subalgebra $\mathscr{A}_{\infty}$ of the algebra $\mathscr{B}_{\infty}$ of partial permutations. This combinatorial interpretation of the symbols $\Sigma_{\mu}$ gives at least the terms of higher weight or higher Kerov degree of a product $\Sigma_{\mu} \Sigma_{\nu}$. In particular, if $k$ and $l$ are bigger than 2, then:

$$
\begin{aligned}
\Sigma_{k} \Sigma_{l} & =\Sigma_{k, l}+\sum_{r \geq 1} \sum_{\begin{array}{c}
a_{1}+\cdots+a_{r}=k \\
b_{1}+\cdots+b_{r}=l
\end{array}} \frac{k l}{r} \Sigma_{\left(a_{1}+b_{1}-1\right), \ldots,\left(a_{r}+b_{r}-1\right)}+\left\{\begin{array}{c}
\text { observable of weight } \\
\text { smaller than } k+l-2
\end{array}\right\} \\
& =\Sigma_{k, l}+\delta_{k, l} k \Sigma_{1^{k}}+\left\{\begin{array}{l}
\text { observable of Kerov } \\
\text { degree smaller than } k+l-1
\end{array}\right\}
\end{aligned}
$$

These expansions will be very useful for proving that renormalized characters are asymptotically gaussian under Schur-Weyl or Gelfand measures, and for computing the limit covariances.

In $\$ 1$, we have computed the expectations of the observables $\Sigma_{\mu}$ under Schur-Weyl and Gelfand measures. Equation (8) implies that $\mathbb{S W}_{n, c}[f]=O\left(n^{\mathrm{wt}(f) / 2}\right)$ for any observable $f$; similarly, (11) implies that $\mathbb{G}_{n}[f]=O\left(n^{\operatorname{deg}_{K}(f) / 2}\right)$ for any $f \in \mathscr{O}$. Using this with $f=\Sigma_{k}$ or $\left(\Sigma_{k}\right)^{2}$, one then gets by Bienaymé-Chebyshev inequality:

$$
\forall k \geq 1, \frac{\Sigma_{k}(\lambda)}{n^{\frac{k+1}{2}}} \longrightarrow \mathbb{S W}_{n, c} c^{k-1} \quad ; \quad \forall k \geq 1, \frac{\Sigma_{k}(\lambda)}{n^{\frac{k+1}{2}}} \longrightarrow_{\mathbb{G}_{n}} \delta_{k, 1}
$$

where the long right arrows indicate convergence in law (here, we have even convergence in probability). Moreover, a change of variables shows that if $\lambda^{*}$ is the scaled version of a Young diagram $\lambda$, then $\widetilde{p}_{k}(\lambda)=$ $n^{k / 2} \widetilde{p}_{k}\left(\lambda^{*}\right)$ for any $k \geq 2$. Since $\Sigma_{k}$ is an observable of weight $k+1$, one has as a consequence $\Sigma_{k}(\lambda)=n^{(k+1) / 2} \Sigma_{k}\left(\lambda^{*}\right)+O\left(n^{k / 2}\right)$, and $[22)$ becomes

$$
\forall k \geq 1, \Sigma_{k}\left(\lambda^{*}\right) \longrightarrow \mathbb{S W}_{n, c} c^{k-1} \quad ; \quad \forall k \geq 1, \Sigma_{k}\left(\lambda^{*}\right) \longrightarrow \mathbb{G}_{n} \delta_{k, 1} .
$$

Finally, (17) allows to recover the limits of the $\widetilde{p}_{k}\left(\lambda^{*}\right)$ 's:

$$
p_{k}\left(\lambda^{*}\right) \longrightarrow \mathbb{W}_{n, c} \sum_{i=1}^{\left\lfloor\frac{k}{2}\right\rfloor} \frac{k^{\downarrow 2 i}}{(k-i) i ! i-1 !} c^{k-2 i} \quad ; \quad p_{k}\left(\lambda^{*}\right) \longrightarrow \mathbb{G}_{n} \begin{cases}\left(\begin{array}{c}
k \\
k / 2
\end{array}\right) & \text { if } k \text { is even, } \\
0 & \text { if } k \text { is odd. }\end{cases}
$$

For continuous Young diagrams, convergence of all the observables (or equivalently, convergence of all the interlacing moments) is equivalent to the uniform convergence on $\mathbb{R}$. As a consequence, (24) implies the existence of limit shapes $\Omega_{c}$ under Schur-Weyl measures and $\Omega=\Omega_{0}$ under Gelfand measures. A lengthy calculation gives an explicit formula for these limit shapes, see [Bia01, p. 8]; in particular,

$$
\Omega_{c}^{\prime}(s \in[c-2, c+2])=\frac{2}{\pi} \arcsin \left(\frac{s+c}{2 \sqrt{1+s c}}\right) \quad ; \quad \Omega^{\prime}(s \in[-2,2])=\frac{2}{\pi} \arcsin \left(\frac{s}{2}\right) .
$$

The previous computations illustrate the use of observables of diagrams in the setting of asymptotic representation theory; for further details, we refer to [IO02]. It turns out that the same methods allow to treat the second order asymptotics, that is to say the asymptotics of fluctuations $\Delta_{n, c}(s)$ or $\Delta_{n}(s)$. Hence, in $\$ 3$, we will show that the observables $\Sigma_{k}$ under Schur-Weyl or Gelfand measures converge in joint law towards a gaussian vector with explicit covariance matrix. Then, in $\$ 4$ by using the combinatorics of $\mathscr{O}$, we will obtain the asymptotic gaussian behaviour of the fluctuations of the shapes of the random partitions. 


\section{Limiting distributions of the renormalized characters}

The algebraic counterpart of Theorem 1 is the following result: under Plancherel measures, rescaled characters values $\Sigma_{k}(\lambda) / n^{k / 2}$ with $k \geq 2$ converge jointly towards independent variables $\sqrt{k} \xi_{k}$, where the $\xi_{k}$ 's are standard gaussian variables of mean 0 and covariance 1 . There is an analoguous result for Schur-Weyl and Gelfand measures, that can be stated as follows:

Theorem 2 We fix a family of independent standard gaussian variables $\left(\xi_{k}\right)_{k \geq 2}$. As $n$ goes to infinity, under Schur-Weyl measures $\mathbb{S W}_{n, c}$, the rescaled character values $X_{k, n, c}=\frac{\Sigma_{k}(\lambda)}{n^{k / 2}}-c^{k-1} n^{1 / 2}$ converge in finite-dimensional laws towards the gaussian variables

$$
X_{k, \infty, c}=\sum_{r=0}^{k-2}\left(\begin{array}{l}
k \\
r
\end{array}\right) c^{r} \sqrt{k-r} \xi_{k-r} .
$$

Similarly, under Gelfand measures $\mathbb{G}_{n}$, the rescaled character values $Y_{k, n}=\frac{\Sigma_{k}(\lambda)}{n^{k / 2}}$ converge in joint law towards the gaussian variables

$$
Y_{k, \infty}=e_{k}+\sqrt{2 k} \xi_{k}
$$

where $e_{k}=0$ if $k$ is even, and $e_{k}=1$ if $k$ is odd.

In the following, $\mathfrak{Q}_{r}$ is the set of set partitions of $\llbracket 1, r \rrbracket$, and if $\pi \in \mathfrak{Q}_{r}$ has $\ell(\pi)$ parts, then $\mu(\pi)$ is the Möbius function of $\pi$, that is to say $(-1)^{\ell(\pi)-1}(\ell(\pi)-1)$ !. The joint cumulant of random variables $X_{1}, \ldots, X_{r}$ is defined by

$$
k\left(X_{1}, \ldots, X_{r}\right)=\sum_{\pi \in \mathfrak{Q}_{r}} \mu(\pi) \prod_{\pi_{j} \in \pi} \mathbb{E}\left[\prod_{i \in \pi_{j}} X_{i}\right]
$$

To prove Theorem 2, one can study joint cumulants of rescaled character values: indeed, if all the joint cumulants of order $r \geq 3$ of the coordinates of a random vector converge to 0 , and if there is a finite limit for the cumulants of order 1 and 2, then the random vector converges in law towards a gaussian vector whose coordinates have for means the limiting cumulants of order 1 , and for covariances the limiting cumulants of order 2 . That said, in the context of asymptotic representation theory of symmetric groups, a sufficient condition for the asymptotic gaussian behaviour of rescaled character values has been exhibited by P. Śniady in [Ś 06$]$. Let $\left(\mathbb{P}_{n}\right)_{n \in \mathbb{N}}$ be a family of probability measures on the sets $\mathfrak{P}_{n}$ of integer partitions; we denote by $\mathbb{E}_{n}$ and $k_{n}$ the corresponding expectations and cumulants for observables of diagrams, viewed as random variables if the diagrams are chosen according to the measures $\mathbb{P}_{n}$. Notice that any family of commuting permutations $\sigma_{1}, \ldots, \sigma_{r}$ can also be considered as a family of random variables via the maps $\left(\sigma_{i}, \lambda\right) \mapsto \chi^{\lambda}\left(\sigma_{i}\right)$.

Proposition 3 The following assertions are equivalent:

1. For all positive integers $l_{1}, \ldots, l_{r}$,

$$
k_{n}\left(\Sigma_{l_{1}}, \ldots, \Sigma_{l_{r}}\right) n^{-\frac{l_{1}+\cdots+l_{r}-r+2}{2}}=O(1) .
$$

2. If $\sigma_{l_{1}}, \ldots, \sigma_{l_{r}}$ are disjoint cycles of respective lengths $l_{1}, \ldots, l_{r}$, then

$$
k_{n}\left(\sigma_{l_{1}}, \ldots, \sigma_{l_{r}}\right) n^{\frac{l_{1}+\cdots+l_{r}+r-2}{2}}=O(1)
$$


In that case known as asymptotic factorization property, the following limits, if they exist, are equal:

$$
\begin{aligned}
& c_{l+1}=\lim _{n \rightarrow \infty} n^{-\frac{l+1}{2}} \mathbb{E}_{n}\left[\Sigma_{l}\right]=\lim _{n \rightarrow \infty} n^{\frac{l-1}{2}} \mathbb{E}_{n}\left[\sigma_{l}\right] \\
& v_{k, l}=\lim _{n \rightarrow \infty} k_{n}\left(\Sigma_{k}, \Sigma_{l}\right) n^{-\frac{k+l}{2}}=\lim _{n \rightarrow \infty} k_{n}\left(\sigma_{k}, \sigma_{l}\right) n^{\frac{k+l}{2}}-k l c_{k+1} c_{l+1}+\sum_{\substack{a_{1}+\cdots+a_{r}=k \\
b_{1}+\cdots+b_{r}=l}} \frac{k l}{r} c_{a_{1}+b_{1}} \cdots c_{a_{r}+b_{r}}
\end{aligned}
$$

the second identity being of course related to Equation (20). Then, the scaled and centered character values $\frac{\Sigma_{k}}{n^{k / 2}}-c_{k+1} n^{1 / 2}$ converge in finite-dimensional laws towards a centered gaussian vector of covariance matrix $\left(v_{k, l}\right)_{k, l \geq 2}$.

Because of Equation (6), the Schur-Weyl measures satisfy trivially (30); this fact was already mentioned in [Ś66. Example 6]. The first part of Theorem 2 is therefore a simple consequence of Formula (32) we also needed to perform a Gram-Schmidt orthogonalization. Unfortunately, we do not know whether the Gelfand measures have the asymptotic factorization property; it might be the case, but for instance, given 4 disjoint transpositions $\tau_{1}, \ldots, \tau_{4}$, Equation (10) only ensures that $k_{n}\left(\tau_{1}, \ldots, \tau_{4}\right)$ is a $O\left(n^{-9 / 2}\right)$, and not a $O\left(n^{-5}\right)$ as would be if Condition 30 were satisfied(ii) Hence, extra technology is needed to establish the second part of Theorem 2, although the idea remains the same, namely, showing that all the cumulants of order $r \geq 3$ of the variables $Y_{k, n}$ vanish at infinity.

To begin with, let us notice that it is easy to demonstrate the simple (non-joint) convergence of the $Y_{k, n}$ 's towards gaussian variables of respective means $e_{k}$ and respective variances $2 k$. Indeed, by induction on $m$, one sees from (21) that

$$
\left(\Sigma_{k}\right)^{m}=\sum_{p=0}^{\left\lfloor\frac{m}{2}\right\rfloor} \frac{m !}{m-2 p ! p !}\left(\frac{k}{2}\right)^{p} \Sigma_{1^{p k} k^{m-2 p}}+\left\{\begin{array}{c}
\text { observable of Kerov degree } \\
\text { smaller than } k m-1
\end{array}\right\}
$$

for any $k \geq 2$. As a consequence, all the moments of the variables $Y_{k, n}$ have limits that can be explicitly computed using simply (11), and these limits are exactly the moments of the gaussian variables $Y_{k, \infty}$; since gaussian variables are characterized by their moments, it implies the non-joint convergence in law. Now, to obtain the joint convergence and the asymptotic independence, one has to prove the two following facts:

$$
\begin{aligned}
& \forall r \geq 3, \forall l_{1}, \ldots, l_{r} \geq 2, k_{n}\left(\Sigma_{l_{1}}, \Sigma_{l_{2}}, \ldots, \Sigma_{l_{r}}\right)=o\left(n^{\frac{l_{1}+l_{2}+\cdots+l_{r}}{2}}\right) \\
& \forall k, l \geq 2, \quad(k \neq l) \Rightarrow k_{n}\left(\Sigma_{k}, \Sigma_{l}\right)=o\left(n^{\frac{k+l}{2}}\right)
\end{aligned}
$$

As $\operatorname{deg}_{K}\left(\Sigma_{k \geq 2}\right)=k$ and $\mathbb{G}_{n}[f]=O\left(n^{\operatorname{deg}_{\mathrm{K}}(f) / 2}\right)$ for any observable $f$, the cumulants above are already known to be $O\left(n^{\frac{l_{1}+l_{2}+\cdots+l_{r}}{2}}\right)$ or $O\left(n^{\frac{k+l}{2}}\right)$; hence, one has to gain only one order of magnitude.

\footnotetext{
(ii) In fact, by using a more precise estimate of the expectations, one can show that this joint cumulant is indeed a $O\left(n^{-5}\right)$, but these are heavy computations and we do not know how to generalize them.
} 
In the algebra $\mathscr{O}$, let us define the disjoint product $\bullet$ by $\Sigma_{\mu} \bullet \Sigma_{\nu}=\Sigma_{\mu \sqcup \nu}$; this product is compatible with the weight of observables or with Kerov's degree. Then, one defines the disjoint cumulant and the identity cumulant of observables $X_{1}, \ldots, X_{r}$ as follows:

$$
\begin{aligned}
k^{\bullet}\left(X_{1}, \ldots, X_{r}\right) & =\sum_{\pi \in \mathfrak{Q}_{r}} \mu(\pi) \prod_{\pi_{j} \in \pi} \mathbb{E}\left[\prod_{i \in \pi_{j}} X_{i}\right] \\
k^{\mathrm{id}}\left(X_{1}, \ldots, X_{r}\right) & =\sum_{\pi \in \mathfrak{Q}_{r}} \mu(\pi) \prod_{\pi_{j} \in \pi}^{\bullet}\left[\prod_{i \in \pi_{j}} X_{i}\right]
\end{aligned}
$$

These new quantities enable us to decompose the standard cumulant of observables as a sum over set partitions

$$
k\left(X_{1}, \ldots, X_{r}\right)=\sum_{\pi \in \mathfrak{Q}_{r}} k^{\bullet}\left(k^{\mathrm{id}}\left(X_{i \in \pi_{1}}\right), \ldots, k^{\mathrm{id}}\left(X_{i \in \pi_{s}}\right)\right) ;
$$

see [Ś06, Proposition 13]. In order to gain one order of magnitude in the estimate of $k\left(\Sigma_{l_{1}}, \ldots, \Sigma_{l_{r}}\right)$, one can use this expansion in disjoint cumulants $k_{\pi}^{\bullet}$, and for each $k_{\pi}^{\bullet}$ :

1. either bound the sum of the Kerov degrees of the identity cumulants by $l_{1}+l_{2}+\cdots+l_{r}-1$;

2. or, in case the sum of the Kerov degrees is maximal and equal to $l_{1}+\cdots+l_{r}$, use the properties of the set partition $\pi$ and Equation (11) to get directly a bound on the disjoint cumulant $k_{\pi}^{\bullet}$.

The first method works for almost all set partitions $\pi$, thanks to a combinatorial interpretation of the identity cumulants of $\Sigma_{l}$ 's in the algebra of partial permutations, see [FM10, Lemma 19]; the remaining cases can be worked out by using a kind of Möbius inversion formula. More precisely:

Lemma 4 The total Kerov degree of the identity cumulants involved in a disjoint cumulant $k_{\pi}^{\bullet}$ is smaller than $l_{1}+l_{2}+\cdots+l_{r}-1$, unless $\pi$ has only parts of size 1 and parts $\pi_{j}$ of size 2 such that if $\pi_{j}=\left\{i_{1}, i_{2}\right\}$, then $\Sigma_{l_{i_{1}}}=\Sigma_{l_{i_{2}}}$.

In that case, $k_{\pi}^{\bullet}$ is proportional to a disjoint cumulant $k^{\bullet}\left(\Sigma_{m_{1}}, \ldots, \Sigma_{m_{s}}, \Sigma_{1^{p_{1}}}, \ldots, \Sigma_{1^{p_{t}}}\right)$, and for Gelfand measures, the higher term of this disjoint cumulant vanishes again, because of the following Möbius inversion formula. Let $(1, \ldots, 1,2, \ldots, 2, \ldots, s, \ldots, s)$ be a sequence of $r$ integers with $r_{1} \geq 1$ integers $1, r_{2} \geq 1$ integers 2, etc. If $\pi=\pi_{1} \sqcup \pi_{2} \sqcup \cdots \sqcup \pi_{\ell(\pi)}$ is in $\mathfrak{Q}_{r}$, we denote by $r_{i j}$ the number of integers $i$ that fall in $\pi_{j}$. Suppose that $s \geq 2$. Then, for any function $F$,

$$
\sum_{\pi \in \mathfrak{Q}_{r}}(-1)^{\ell(\pi)-1}(\ell(\pi)-1) ! \prod_{j=1}^{\ell(\pi)} \prod_{r_{i j} \geq 1} F\left(i, r_{i j}\right)=0
$$

The proof of the second part goes by induction on the $r_{i}$ 's. As a consequence of Lemma 4 and of the previous discussion, all the disjoint cumulants in the expansion of a standard cumulant of observables $\Sigma_{l}$ as in (34) or (35) have a smaller order of magnitude, which ends the proof of Theorem 2. As mentioned in the introduction, although Lemma 4 may seem rather innocent and technical, it is a key argument when Proposition 3 fails to apply and one wants nethertheless to establish the asymptotic gaussian behaviour of character values. 


\section{Central limit theorems for the shapes of the random partitions}

Finally, let us explain how to translate Theorem 2 in terms of the fluctuations $\Delta_{n, c}(s)$ and $\Delta_{n}(s)$. It is clear that the moments

$$
\int_{\mathbb{R}}(s-c)^{k}\left[\sqrt{n}\left(\lambda^{*}(s)-\Omega_{c}(s)\right)\right] d s \quad \text { and } \quad \int_{\mathbb{R}} s^{k}\left[\sqrt{n}\left(\lambda^{*}(s)-\Omega(s)\right)\right] d s
$$

can be written in terms of the observables $\widetilde{p}_{k}(\lambda)$; then, using equations (17) or (18), one can express these moments in terms of the $X_{k, n, c}$ 's or the $Y_{k, n}$ 's, up to a negligible remainder. Thus:

$$
\begin{aligned}
\left\langle(s-c)^{k} \mid \Delta_{n, c}\right\rangle & \simeq_{\mathbb{S W}_{n, c}} \frac{2}{k+1} \sum_{l=0}^{k-1}\left(\sum_{m=0}^{\left\lfloor\frac{l}{2}\right\rfloor}\left(\begin{array}{c}
k+1 \\
m
\end{array}\right)\left(\begin{array}{c}
k+1-2 m \\
l-2 m
\end{array}\right)(-c)^{l-2 m}\right) X_{k+1-l, n, c} \\
\left\langle s^{k} \mid \Delta_{n}\right\rangle & \simeq_{\mathbb{G}_{n}} \frac{2}{k+1} \sum_{m=0}^{\left\lfloor\frac{k-1}{2}\right\rfloor}\left(\begin{array}{c}
k+1 \\
m
\end{array}\right) Y_{k+1-2 m, n}
\end{aligned}
$$

Then, it has been shown in [IO02] that these identities can be reversed by using the Chebyshev polynomials of the second kind $U_{k}(X)$, renormalized so that $U_{k}(2 \cos \theta)=\frac{\sin (k+1) \theta}{\sin \theta}$. Hence:

$$
\begin{aligned}
\left\langle U_{k}(s-c) \mid \Delta_{n, c}\right\rangle & \simeq_{\mathbb{S W}_{n, c}} \frac{2}{k+1} \sum_{l=0}^{k-1}\left(\begin{array}{c}
k+1 \\
l
\end{array}\right)(-c)^{l} X_{k+1-l, n, c} \longrightarrow \mathbb{S W}_{n, c} 2 \sqrt{\frac{1}{k+1}} \xi_{k+1} \\
\left\langle U_{k}(s) \mid \Delta_{n}\right\rangle & \simeq_{\mathbb{G}_{n}} \frac{2 Y_{k+1, n}}{k+1} \longrightarrow \mathbb{G}_{n} \frac{2 e_{k+1}}{k+1}+2 \sqrt{\frac{2}{k+1}} \xi_{k+1}
\end{aligned}
$$

The calculations are really the same as in [IO02, §7] for Gelfand measures; on the other hand, they are much more convoluted in the case of Schur-Weyl measures, and they involve various hypergeometric identities (actually, it is quite a miracle to obtain at the end such a simple expression for $\left\langle U_{k}(s-c) \mid \Delta_{n, c}\right\rangle$ ). As the Chebyshev polynomials $U_{k}$ form an orthogonal basis, the same discussion as in [IO02, §9] gives us finally the convergence in law of the deviations in the space of distributions:

Theorem 5 In the sense of distributions on the interval $[c-2, c+2]$, under the Schur-Weyl measures $\mathbb{S} W_{n, c}, \Delta_{n, c}(s)$ converges in law towards the generalized gaussian process

$$
\Delta(s-c),
$$

where $\Delta$ is as in Theorem [1] Similarly, in the sense of distributions on the interval $[-2,2]$, under the Gelfand measures $\mathbb{G}_{n}, \Delta_{n}(s)$ converges in law towards the generalized gaussian process

$$
\frac{1}{2}-\frac{\sqrt{4-s^{2}}}{\pi}+\sqrt{2} \Delta(s)
$$

The precaution "in the sense of distributions" is justified, because the infinite sum of random variables $\Delta(s)$ does not converge pointwise. However, it makes sense as a distribution - this is the same phenomenon as for the well-known gaussian free field. It is really an amazing fact that the same gaussian process is involved in the asymptotics of Plancherel, Schur-Weyl and Gelfand measures. Let us conclude our extended abstract with an open problem. We define the $\boldsymbol{\beta}$-Plancherel probability measures by: 


$$
\mathbb{P}_{n, \beta}[\lambda]=\frac{\left(\operatorname{dim} S^{\lambda}\right)^{\beta}}{\sum_{\mu \in \mathfrak{P}_{n}}\left(\operatorname{dim} S^{\mu}\right)^{\beta}}, \quad \beta>0 .
$$

They are discrete analogues of the $\beta$-ensembles of random matrices (see [BR01]), and when $\beta=1$ or 2 , one recovers the Gelfand measures and the Plancherel measures. One can conjecture that under $\beta$ Plancherel measures, the fluctuations $\sqrt{n}\left(\lambda^{*}(s)-\Omega(s)\right)$ converge in law towards generalized gaussian processes, possibly of the form

$$
f_{\beta}(s)+\sqrt{\frac{2}{\beta}} \Delta(s)
$$

with $f_{\beta}$ deterministic function on $[-2,2]$. It would be interesting to prove such a general result.

\section{References}

[APR08] R. M. Adin, A. Postnikov, and Y. Roichman. Combinatorial Gelfand models. J. Algebra, 320(3):1311-1325, 2008.

[Bia01] P. Biane. Approximate factorization and concentration for characters of symmetric groups. Internat. Math. Res. Notices, 4:179-192, 2001.

[BR01] J. Baik and E. Rains. The asymptotics of monotone subsequences of involutions. Duke Math. J., 109(2):205-281, 2001.

[FM10] V. Féray and P.-L. Méliot. Asymptotics of q-Plancherel measures. To appear in Probability Theory and Related Fields, arXiv 1001.2180v1 [math.RT], 2010.

[Ful97] W. Fulton. Young Tableaux with Applications to Representation Theory and Geometry, volume 35 of London Mathematical Society Students Texts. Cambridge University Press, 1997.

[IK99] V. Ivanov and S. Kerov. The algebra of conjugacy classes in symmetric groups, and partial permutations. In Representation Theory, Dynamical Systems, Combinatorial and Algorithmical Methods III, volume 256 of Zapiski Nauchnyh Seminarov POMI, pages 95-120, 1999.

[IO02] V. Ivanov and G. Olshanski. Kerov's central limit theorem for the Plancherel measure on Young diagrams. In Symmetric Functions 2001: Surveys of Developments and Perspectives, volume 74 of NATO Science Series II. Mathematics, Physics and Chemistry, pages 93-151, 2002.

[LS77] B. F. Logan and L. A. Shepp. A variational problem for random Young tableaux. Adv. Math., 26:206-222, 1977.

[Mél10a] P.-L. Méliot. Asymptotics of the Gelfand models of the symmetric groups. arXiv $1009.4047 \mathrm{v} 1$ [math.RT], 2010.

[Mél10b] P.-L. Méliot. Kerov's central limit theorem for Schur-Weyl measures of parameter $\alpha=1 / 2$. arXiv 1009.4034v1 [math.RT], 2010.

[Ś06] P. Śniady. Gaussian fluctuations of characters of symmetric groups and of Young diagrams. Probability Theory and Related Fields, 136(2):263-297, 2006. 